Running Head: INTERFERENCE AND PROBLEM SIZE EFFECT

\title{
TITLE
}

\section{Disentangling neural sources of problem-size and interference effects in multiplication}

Kerensa tiberghien ${ }^{1}$, Muhammet I. Sahan ${ }^{1}$, Bert De Smedt², Wim Fias ${ }^{1}$, Ian M. Lyons ${ }^{3}$

${ }^{1}$ Ghent University, Department of Experimental Psychology

2University of Leuven, Parenting and Special Education Research Unit

${ }^{3}$ Georgetown University, Psychology Department

Funding Information: This research was supported by Interuniversity Attraction Poles Program of the Belgian Federal Government (P7/11), Ghent University (GOA 01G01108) to Fias, Belgian Fund for Scientific Research - Flanders (project G063817N) to De Smedt and Fias, Banting Postdoctoral Fellowship to Lyons (National Sciences and Engineering Research Council, Canada), Departmental Start-Up Funds to Lyons (Georgetown University, Psychology Department) Travel Grant Faculty Mobility Fund Ghent University to Lyons. The authors declare no financial conflicts of interest. 


\begin{abstract}
Multiplication is thought to be primarily solved via direct retrieval from memory. Two of the main factors known to influence the retrieval of multiplication facts are problem-size and interference. Because these factors are often intertwined, we sought to investigate the unique influences of problem-size and interference on both performance and neural responses during multiplication fact-retrieval in healthy adults. Behavioral results showed that both problem-size and interference explained separate unique portions of response-time variance, but with significantly stronger contribution from problem-size, which contrasts with previous work in children. Whole-brain fMRI results relying on a paradigm that isolated multiplication fact-retrieval from response-selection showed highly overlapping brain areas parametrically modulated by both problem-size and interference in a large network of frontal, parietal and subcortical brain areas. Subsequent analysis within these regions revealed problem-size to be the stronger and more consistent unique modulating factor in overlapping regions as well as those that appeared to respond only to problem-size or interference at the whole-brain level, thus underscoring the need to look beyond anatomical overlap using arbitrary thresholds. Additional unique contributions of interference (beyond problem-size) were identified in right angular gyrus and subcortical regions associated with procedural processing. Together, our results suggest that problem-size, relative to interference, tends to be the more dominant factor in driving behavioral and neural responses during multiplication fact retrieval in adults. Nevertheless, unique contributions of both factors demonstrate the importance of considering the overlapping and unique contributions of each in explaining the cognitive and neural bases of mental multiplication.
\end{abstract}




\section{INTRODUCTION}

Arithmetic is a quintessential mathematical ability, serving in many ways as a cornerstone of everyday numeracy skills (Ashcraft \& Guillaume, 2009; Campbell, 2005). Memory retrieval has long been understood to play a key role in arithmetic processing, and in particular, in retrieving multiplication facts (e.g., Campbell \& Xue, 2001; Kirk \& Ashcraft, 2001; Fayol \& Barrouillet, 2002). Two of the main factors thought to influence multiplication memory retrieval are problem-size (e.g., Stazyk, Ashcraft, \& Hamann, 1982) and interference (De Visscher \& Noël, 2014a, 2014b; De Visscher et al., 2015), both on a behavioral and neural level. However, these two factors are often inter-twined, and as such, the unique influences of both problem-size and interference on memory representation remain poorly understood, particularly at the neural level.

Among the factors thought to influence multiplication memory retrieval, the problem-size effect has perhaps received greatest interest. The problem-size effect (PSE) is a robust finding, wherein poorer performance (slower and more error prone) is typically observed for larger problems relative to smaller problems. Different explanations of the PSE have been suggested. A leading explanation for the PSE suggests that the frequency with which arithmetical problems are taught in school impacts how a given item is stored or represented in memory (Ashcraft, 1987; Ashcraft \& Christy, 1995; McCloskey \& Lindemann, 1992). Large problems are encountered less frequently and are therefore stored in memory with lower strength, resulting in poorer performance relative to small problems. Similarly, Siegler and Shrager (1984) suggested that learned problems are associated with correct and incorrect answers. Small problems have a lesser history of error, leading to only weak associations with erroneous answers, in turn leading to a high likelihood of retrieving the correct answer. On the other hand, large problems have a larger history of errors because of the possibility of making errors during the execution of calculation procedures (e.g. 6x7 = $7+7+7+7+7+7$ ) at early stages of learning. Consequently, the strength of the association from a large problem to an incorrect answer is relatively high, compared to the strength of small problems. Another explanation for the PSE is put forward by Verguts and Fias's (2005) model of interacting neighbors. Within this model, the PSE can be understood from the principles of cooperation and competition. When presenting a problem in an associative network, neighboring problems can either cooperate (if they lead to the 
same response, because they share the decade or unit; e.g., $4 \times 6=24 \& 4 \times 7=28$ ) or compete (if they lead to a different response, because they do not share the decade or unit; e.g., $4 \times 4=16 \& 4 \times 5=20$ ). When neighboring problems lead to the same response, they are consistent and hence facilitate the retrieval of the correct answer, whereas inconsistent neighbors will compete with one another and therefore interfere with the retrieval of the correct answer.

Recently, another memory effect has been described that modulates performance of multiplication facts - namely, the interference effect. The multiplication interference effect was first described by De Visscher and Noël (2014a) and is based on feature overlap theory (Nairne, 1990; Oberauer \& Lange, 2008), which proposes interference arises directly as a function of the number of overlapping features between two problems. Thus, when more recently learned problems have to be retrieved, they will be subject to proactive interference as a function of the number of overlapping features with previously learned problems. Because multiplication tables tend to be learned sequentially from the $2 \mathrm{x}$ times-table to the $3 \mathrm{x}$ times-table, up to the $9 \mathrm{x}$ times-table, and so on, it has been suggested that multiplication tables are subject to proactive interference during learning. This interference results in a gradual decrease of memory encoding weight as operand quantities increase. To test this idea, De Visscher and Noël (2014a) developed a measure of the overlap of the proactive interference between multiplication facts, which they termed an interference parameter. The interference parameter represents the degree of proactive interference by calculating the digit overlap between a given problem (including the solution) with previously learned problems. In this way, each problem is assigned 'proactive interference points'. One point is given when there is an overlap of 2 digits (e.g., $\underline{2} \times \underline{8}=16$ and $\underline{2} \times 4=\underline{8}$ ), while three points are given when 3 digits overlap (e.g., $\underline{3} \times 9=\underline{27}$ and $\underline{3} \times \underline{7}=\underline{2} 1$ ). For example, the interference parameter of $3 \times 6=18$ is 8 because the proactive interference comes from 6 previously learned problems $(2 \times \underline{3}=\underline{6}, 2 \times \underline{6}=\underline{12}, 2 \times \underline{8}=\underline{16}, \underline{3} \times 2=\underline{6}, \underline{3} \times 4=\underline{12}, \underline{3} \times 5=\underline{15})$. The study showed that problems with a higher interference parameter yielded longer reaction times (see also De Visscher \& Noël, 2014b).

It is important to point out that problem-size and proactive interference have similar predicted effects on performance: larger problem-size should lead to poorer performance, and larger problems involve higher times-tables and so, due to increased proactive interference, should also lead to poorer performance. This means that 
problem-size and interference effects are strongly correlated (indeed, $r=.55$ for operands 2 through 9; De Visscher \& Noël, 2014a,b). In fact, the network model of arithmetic proposed by Campbell (1995) explicitly posits interference as a key mechanism to explain the problem-size effect. Specifically, large problems are associated with a wider range of other problems (relative to smaller problems), which induces a higher degree of retrieval interference (and hence poorer performance on large problems). An important question is thus whether interference and problem-size effects are essentially different descriptors of the same underlying mechanism, or whether the two factors might be separated into distinct - i.e., unique - contributions with respect to arithmetic processing.

To this end, De Visscher et al., (2016) directly compared the predictive capacity of problem-size and interference parameters on typed multiplication production response-times in $4^{\text {th }}$ grade children. At the individual subject level, unique effects of both parameters were found (albeit somewhat stronger for interference: $t=4.4$ vs 2.5); at the sample level (averaging response-times across all children for a given trial), the authors found that only the interference parameter captured significant unique variance. Together, the De Visscher et al. results suggest that interference is the stronger determinant of multiplication fact retrieval performance in children.

A few questions remain, however. First, De Visscher et al. (2016) examined children still acquiring basic arithmetic knowledge. One question is thus whether interference or problem-size is the stronger predictor of multiplication performance in adults whose fact-retrieval is more likely to be heavily practiced and thus more efficient. Second, De Visscher et al. used a typing production task (children typed their answers); because the interference parameter is directly related to the number of overlapping digits, this may have inflated the relation with multiplication response-times as answers requiring the same digits to be typed would likely yield similar response-times. Third, it would be potentially informative to examine the relative contributions of problem-size and interference to predicting neural responses during retrieval of multiplication facts. Moreover, by focusing on just the retrieval phase, fMRI has the potential to isolate computation from response selection, thus mitigating the potential influence of extraneous aspects of the task not of immediate theoretical interest.

In an fMRI study, De Visscher et al. (2015) assessed neural responses while participants verified whether multiplication problems had been correctly solved. To 
isolate problem-size and interference, the authors chose a subset of problems in order to fill out an orthogonal 2(problem-size: small, large) $\times 2$ (interference: high, low) design.

Results showed that most regions sensitive to one effect were also sensitive to the other (two main effects); indeed no interactions were found at the whole-brain level. The authors did find that left angular gyrus (LAG) was sensitive to interference but not problem-size and the opposite pattern in right intraparietal sulcus (RIPS; see also, De Visscher et al., 2018).

These two fMRI studies (De Visscher et al., 2015, 2018) converge to show broadly overlapping effects for problem-size and interference at the neural level, with some evidence indicating the two effects may be dissociable as well. However, due to the ANOVA-based design, neither study was able to quantify the relative degree of unique and overlapping contribution of each parameter to modulation of neural responses. Only orthogonal components could be identified, and this was specific to a highly selected subset of problems, thus obfuscating the natural correlation between the two parameters when considering multiplication processing more generally. Instead, a parametric fMRI design similar to that used in assessing the relative contributions of the two parameters to behavioral responses (e.g., De Visscher et al., 2016) might be more optimal for this purpose. Furthermore, with respect to both studies (De Visscher et al., 2015,2018 ), two additional issues are worth noting: (1) Both used a verification procedure wherein the presence of the proposed solution can alter the nature of retrieval processing (e.g., by a priori narrowing the retrieval search space); and (2) both studies modeled activity across retrieval and response selection which introduces confounds from the presence and need to process the verification stimulus and the selection of a specific motoric response. FMRI provides the opportunity to separate computation from response selection, which may be particularly crucial in the current case given that interference and problem-size may interact differently with response selection than with retrieval processing.

In the present study, we used a parametric approach to disentangle the overlapping and unique influences of problem-size and interference on behavioral performance and neural response patterns in healthy adults. Specifically, we used a parametric univariate approach for the neural data, wherein we identified brain regions (via whole-brain analysis) whose responses were systematically modulated by interference, problem-size, or both. Within these regions, we then assessed the extent to 
which each factor - interference or problem-size - uniquely explained the whole-brain result. We did so via a univariate multiple regression approach (similar to that used for the behavioral data). In this way, we provide a systematic assessment of the relative unique contributions of interference and problem-size to multiplication fact retrieval in adults at both the behavioral and neural levels. Moreover, the fMRI paradigm we used was designed to isolate multiplication fact-retrieval from response selection, and the behavioral paradigm relied on verbal responses rather than typed responses; together, these two methodological aspects of our approach allowed us to reduce the likelihood that response-related factors may have driven our results. In sum, our aim was to quantify the respective roles of problem-size and interference on multiplicationretrieval via behavioral and neural measures in healthy adults with mature mental arithmetic systems.

\section{METHODS}

\section{Participants}

Thirty adults from Ghent University participated in the experiment (22 female, mean age=24yrs, range: 18-27yrs, all right-handed). All participants had normal or correctedto-normal vision and reported no history of neurological or psychiatric illness. Prior to taking part in the study, participants gave written consent. All participants were paid $€ 40$ for their participation. The study was approved by the Medical Ethical Committee of Ghent University and Ghent University Hospital. Six participants were excluded from further analyses ( 4 due to excessive movement, 1 due to technical difficulties, 1 was diagnosed with dyscalculia), leaving a final $N$ of 24 participants.

\section{Procedure}

All experiments were presented via E-Prime (Psychology Software Tools, Pittsburg, PA) and displayed on a $1600 \times 900$ resolution screen. Participants performed an arithmetic task both prior to and during scanning. For the behavioral task, the computer was placed on average $50 \mathrm{~cm}$ in front of the participant. In the scanner, stimuli were presented via a Brainlogics 200MR digital projector visible via a mirror attached to the head coil, with a viewing distance of $120 \mathrm{~cm}$. 


\section{Tasks}

We should note that the data reported here are part of a larger dataset; all results reported here are unique and address hypotheses that do not overlap with any other current or future publications arising from this dataset. In particular, both the pre-scan and fMRI arithmetic tasks included three operations: Multiplication, Addition and Subtraction. Operation order was fully randomized across participants, so the presence of subtraction problems should not yield systematic biases when considering just the multiplication and addition problems. Here we limit our attention to just Multiplication problems as these are directly relevant to the hypotheses of theoretical interest here (i.e. memory retrieval). Because, to date, prior work on interference effects have been examined almost exclusively in multiplication, we were primarily interested in investigating the unique contribution of the problem-size effect and interference effect on multiplication.

\section{Pre-Scan Arithmetic Task}

The arithmetic task was a production task (i.e., task where the participant needed to generate the answer) containing all permutations of two operands ranging from 0 to 10 (121 total problems) with three different arithmetic operations: addition, multiplication and subtraction (resulting in a grand total of 363 problems). All problems were presented once, with order randomized across participants. In keeping with prior work (e.g., De Visscher et al., 2016), we focused on multiplication problems from $2 \times 2$ to $9 \times 9$, though notably, here we have 64 trials per subject in this range (as opposed to 36 in De Visscher et al.), which should allow for more accurate assessment of problem-size and interference effects at the individual subject level. Note that the multiplication tables 0 , 1 and 10 were not included. The reason is that these problems are probably solved by means of rule-based strategies (e.g., Sokol, McCloskey, Cohen, \& Aliminosa, 1991) and hence the problem-size and interference effects may not necessarily apply for these multiplication problems.

A trial started with a fixation (three squares) presented for $3000 \mathrm{msec}$ followed by the arithmetic problem. The problem remained on the screen until the participant responded. Once the participant said the response out loud, a voice-key recorded the onset of speech. Next, the experimenter recorded the participant's response and noted if the voice-key triggered correctly. For instance, a participant might occasionally extend a response over a long duration, or they may have unwittingly made an extraneous sound 
such as "uhm" or cough that accidentally triggered the voice-key. On such trials, the data were discarded, and that same problem was presented again at a randomly chosen time later in the pre-scan task. The inter-trial-interval was $1000 \mathrm{msec}$. This procedure was maintained, until a valid response was recorded for all 363 problems Short breaks were given after every 33 trials.

\section{Arithmetic Task-fMRI Version}

The arithmetic task inside the scanner was kept as similar to the pre-scan version as possible: all problems ranging from 0 to 10 were used in addition, multiplication and subtraction, resulting in a grand total of 363 problems. Trials were divided as evenly as possible across 6 separate runs, with trial (and hence also run) order randomized across participants. As in the pre-scan task, a trial started with a fixation presented for $3000 \mathrm{msec}$ followed by the first arithmetic problem. Here, the problem (e.g., $9 \times 8$ ) remained on the screen for $2600 \mathrm{msec}$. Participants were instructed to mentally compute the answer during this period. For most trials, the problem was then replaced by fixation. For 10 percent of trials, a response was required, in which case the problem was replaced by two response possibilities for $1500 \mathrm{msec}$. One number was the correct response, while the other number was the correct response $+/-1$. Participants pressed either a left or a right key (left or right index finger) to indicate the correct answer. Inter-trial interval (fixation) was jittered (range $=1000-8194 \mathrm{msec}$, mean $=3421 \mathrm{msec}$ ) for all trials. Response events were modeled as events of no interest.

It is important to note that, in the scanner, the focus was less on response times and more on participants' mental calculation of the solution. That is, our goal was to separate the mental calculation aspects of arithmetic processing from response preparation and execution. Therefore, participants were instructed to mentally compute the answer to each problem while it remained on the screen, without an overt response. Responses were therefore probed on only 10 percent of all problems. These response probes were randomly assigned to different problems (thus, this randomization was different for each participant). Hence, participants could not predict in advance which problems would require a response, and the association between a response event and a given problem was thus not systematic across participants. Accuracy for problems of the scanner-task was high $(M=93.9 \%, S E=1.5 \%)$ and highly similar to problems outside the scanner (pre-scan task; $M=93.9 \%, S E=0.8 \%$ ). Average response-times were faster than those seen for the pre-scan behavioral task (pre-scan: $M=1150 \mathrm{msec}, S E=61 \mathrm{msec}$; in- 
scan: $M=681 \mathrm{msec}, S E=19 \mathrm{msec}$ ), which is what one would expect if participants were computing the answer during presentation of the problem $(2600 \mathrm{msec})$ prior to appearance of the (occasional) verification probe. Further evidence that participants were engaging with the task as instructed is the presence of problem-size and interference effects in the neural data (see Results). That is, fMRI analyses focused exclusively on the calculation period (prior to response). It is difficult to explain how a problem-size or interference effect could have been observed during this period if participants were simply ignoring problem presentation and waiting until the sporadic presentation of response options to engage with the task.

\section{fMRI Data Acquisition and Preprocessing}

Images were collected with a 3T Siemens Magnetom Trio MRI system (Siemens Medical Systems, Erlangen, Germany) using a 32-channel radio frequency head coil. Participants were positioned headfirst and supine in the magnet bore. Subjects were instructed to move their heads as little as possible throughout the entire scanning session. A wholebrain high-resolution anatomical scan was acquired using a standard 3D MPRAGE sequence (voxel size $=1 \mathrm{~mm}^{3}$ ). Functional images were collected using an echo-planar imaging (EPI) sequence: $\mathrm{TR}=2600 \mathrm{msec}, \mathrm{TE}=28 \mathrm{msec}$, flip angle $=80^{\circ}$; in-plane matrix of $3.3 \mathrm{~mm}^{2}$ voxels $=64 \times 64(\mathrm{FOV}=211 \mathrm{~mm})$, with slice thickness $=3.3 \mathrm{~mm}$ (44 slices, interleaved, no skip), yielded $3.3 \mathrm{~mm}^{3}$ isometric voxels.

Structural and functional images were analyzed using Brain Voyager QX 20.4 (Brain Innovation, Maastricht, Holland). Functional data were interpolated to $3 \mathrm{~mm}^{3}$ and corrected for slice scan-timing using cubic spline interpolation, corrected for headmotion (trilinear/sinc interpolation), and finally high-pass filtered using a GLM procedure with a Fourier basis set. Excessive motion was deemed net drift $>3 \mathrm{~mm}$ in a given run or $>1.5 \mathrm{~mm}$ sudden movement; participants with runs exceeding these criteria were removed from analysis $(n=4)$. Participants' functional images were then coregistered to their respective anatomical scans using 12-parameter gradient-based affine alignment, and anatomical images were co-registered into Talairach space (Talairach \& Tournoux, 1988). For univariate analyses, functional data were spatially smoothed at 3mm FWHM; for multivariate (RSA) analyses, unsmoothed data were used. Multivariate analyses were conducted using Matlab (2016a). 


\section{Analysis Approach}

\section{Problem-Size}

The problem-size can be measured with several indices (i.e., minimum operand, maximum operand, sum, sum squared, product). However, the product of the two operands is more frequently used (Campbell, 1997). For that reason, and because the product was a better predictor of performance in the study by De Visscher and Noël (2014), we have used the product as measure of the problem-size (see Appendix A). An important difference is that our study has included the commutative pairs (e.g., $2 \times 4$, $4 \times 2)$ whereas the study of De Visscher and Noël (2014; 2015) did not include them, which increased the available number of trials per participant from 36 to 64 , thereby increasing the precision with which parametric effects of problem-size and interference could be estimated for each participant.

\section{Interference}

As noted in the introduction, the interference parameter is based on the feature overlap theory (Nairne, 1990; Oberauer \& Lange, 2008). The idea is that problems that are similar to previously encoded problems will be recalled more poorly due to proactive interference. Hence, similarity can be quantified by the feature overlap between problems. Multiplication tables also contain proactive interference during learning, because these are learned from the two times table up to the nine times table. De Visscher and Noël (2014) calculated the digit overlap (i.e., the proactive interference) between a problem (including its solution) and the previously learned problems, resulting in an interference parameter. The interference parameter is based on 'proactive interference points'. One point is given when there is an overlap of 2 digits (e.g., $\underline{2} \times \underline{8}=16$ and $\underline{2} \times 4=\underline{8}$ ) and three points are given when 3 digits overlap (e.g., $\underline{3} \times$ $9=\underline{27}$ and $\underline{3} \times \underline{7}=\underline{21}$ ). For example, the problem ' $3 \times 6=18$ ' has an interference parameter of 8 , because of proactive interference from 6 previously learned problems $(1$ point for $2 \times \underline{3}=\underline{6}, 2 \times \underline{6}=\underline{12}, \underline{3} \times 2=\underline{6}, \underline{3} \times 4=\underline{12}, \underline{3} \times 5=\underline{15}$ and 3 points for $2 \times \underline{8}=\underline{16}$ ). This was done for every multiplication from $2 \times 2$ to $9 \times 9$, resulting in an interference parameter that ranged from 0 to 25 (See appendix A).

\section{Behavioral Analyses}

Accuracy levels were high both for the pre-scan and in-scanner tasks (94\% in both cases). Such near-ceiling performance levels provide minimal variability for modelfitting; hence, analyses here focus instead on response-times (RTs). The two parameters 
(problem-size and interference) were used to predict RTs, separately for each subject. Each problem ranging from $2 \times 2$ to $9 \times 9$ was assigned a problem-size (based on the product of the operands) and an interference parameter (see above and Appendix A). Parameter fits were estimated in two ways: (1) each predictor treated separately (zeroorder relations) and simultaneously (unique relations). Zero-order and unique $r$-values for each predictor were extracted for each subject, which quantified the degree of unique fit between a given parameter and RT; tests were then conducted on Fisher $z$ transformed $[z=\operatorname{atanh}(r)] r$-values across subjects.

\section{fMRI Analyses}

The same parametric approach was used for fMRI analysis. Predictors comprised trials in the same manner as the behavioral results (other trials and response events were modeled as events of no interest), in order to parallel the behavioral analyses as closely as possible. Note that parametric predictors were scaled from 0 to 1 to make resulting beta-weights more interpretable. A standard voxel-wise GLM was run with parametric predictors for Problem-Size and Interference, in addition to the main effect of Multiplication (i.e., a single predictor with all multiplication trials weighted equally). Due to the high degree of collinearity between the parametric predictors, if run together in the same GLM, the beta weights associated with Problem-Size and Interference would each carry very large error estimates, making their interpretation problematic. Thus, at the whole-brain-level, we ran two separate GLMs: each containing just one of the parametric predictors. Note that this also allowed us, initially at least, to identify regions sensitive to each effect separately. ${ }^{1}$ For each GLM, ROIs were identified via a conjunction with the overall effect of multiplication: Problem-Size: (Multiplication $\cap$ Problem-Size), Interference: (Multiplication $\cap$ Interference). A given region, was thus responsive to multiplication processing in general, and it was modulated systematically as a function of problem-size, for instance. Resulting statistical maps were thresholded using an uncorrected voxel-wise threshold of $p<.001,2$ and subsequently cluster-

\footnotetext{
${ }^{1}$ One can think of these initial whole-brain results as akin to zero-order correlations: that is, they quantify the relation between Problem Size and brain activity, and between Interference and brain activity separately. As much as the zero-order correlation table is useful in understanding behavioral data, understanding the overlapping and non-overlapping ROIs sensitive to Problem Size and Interference is useful in contextualizing the overall pattern of results.

${ }^{2}$ In practice, this meant that each separate map in the conjunction (e.g., main effect of Multiplication and effect of Problem Size) was thresholded at $p<.002$, because the joint probability of two non-independent contrasts obtaining in the same voxel is $1-\sqrt{1-.002}=.001$.
} 
corrected for multiple comparisons using a Monte Carlo simulation procedure (Forman et al., 1995) at $\alpha<.01$.

Within each ROI thus identified, we then partialled out unique modulation of brain activity associated with each parameter, Problem-Size and Interference. This was done by creating residualized versions of each parametric predictor, in that each was residualized with respect to its counterpart. In this way the residualized Problem-Size predictor no longer contained variance associated with Interference, and vice versa. We then computed betas associated with each of these residualized predictors (controlling also for the main effect of Multiplication, as with the whole-brain analyses) separately for each subject in each ROI. Finally, these betas were then contrasted against 0 using a standard $t$-test. In this way, within ROIs that responded to Problem-Size, Interference, or both, we then computed the extent to which this effect was associated uniquely with each factor.

\section{RESULTS}

\section{Behavioral Results}

Behavioral results are summarized in Figure 1. The left side of Figure 1 shows zeroorder fits; the right side shows unique fits (the fit between problem-size and RTs controlling for the interference, and vice versa). Zero-order fits are provided mainly for context. Our primary theoretical interest here is with the unique predictive capacity of each parameter. Both problem-size and interference uniquely predicted longer RTs in that the average (z-transformed) unique correlation value across participants was well above zero [problem-size: $M=.346$, $s e=.029, t(23)=12.00, p=2.2 \mathrm{E}-11$; interference: $M$ $=.194, s e=.027, t(23)=7.15, p=2.7 \mathrm{E}-07$; see also Figure 1, right). Crucially, problemsize captured significantly more unique variance relative to interference ( $p=3.7 \mathrm{E}-04)$.

To summarize, results show that both problem-size and interference predicted performance, and that each contributed unique variance, although problem-size contributed significantly greater unique variance. In the next section, we examine the overlapping and unique contributions of these factors insofar as each predicts neural activity during mental multiplication fact-retrieval, independent of response demands. 


\section{Figure 1: Behavioral Results}

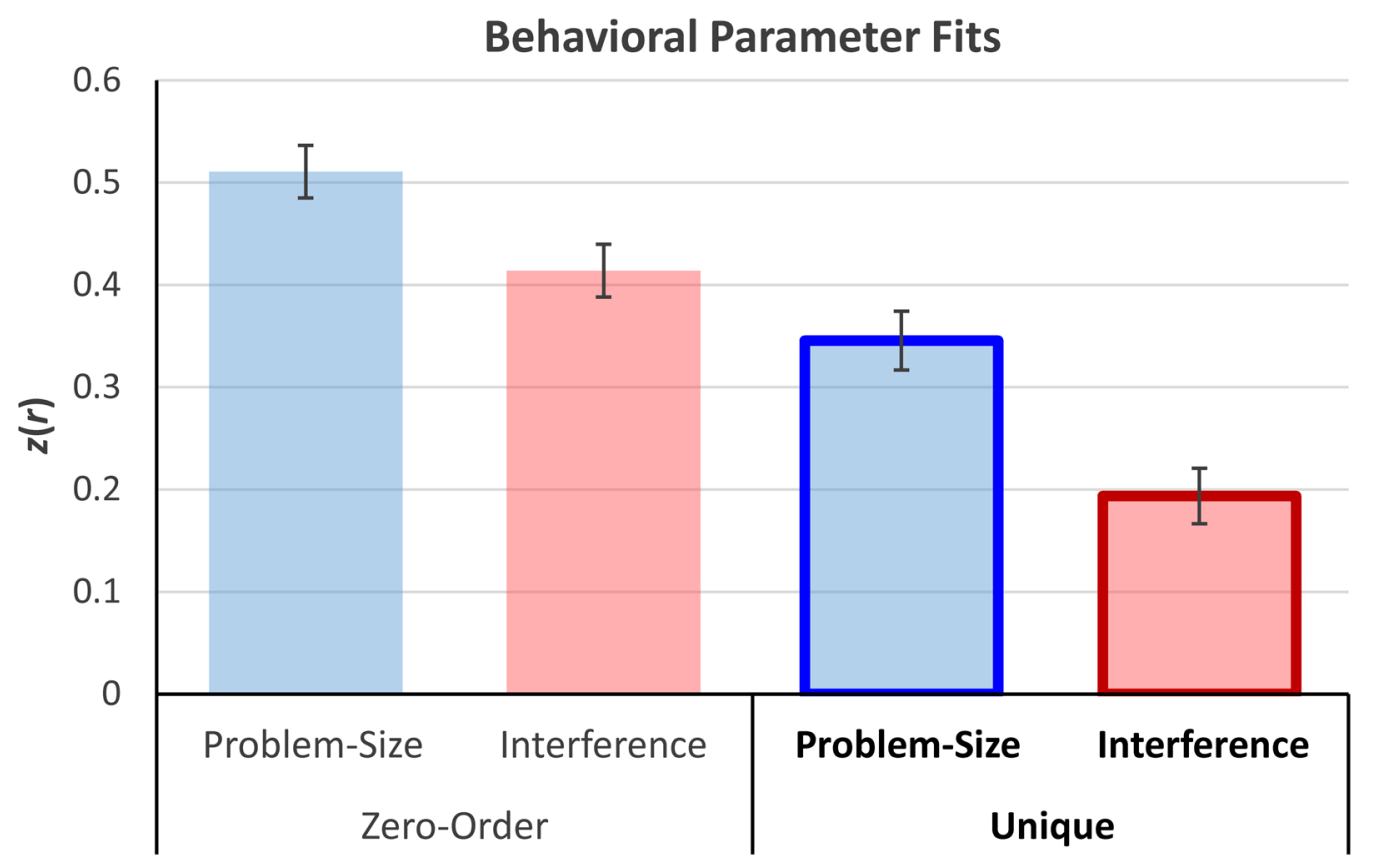

Figure 1 shows zero-order (left) and unique (right) predictive capacities of problem-size and interference (for RTs). Values are Fisher $z$-transformed $r$-values, which were computed for each subject separately, then averaged across subjects. Error-bars are standard-errors of the mean.

\section{fMRI Results}

\section{Whole-Brain Zero-Order Effects}

Whole-brain univariate results are shown in Figure 2, with anatomical details given in

Table 1. Consistent with the notion that Problem-Size and Interference are highly intertwined, the majority of areas sensitive to one parameter were also sensitive to the other (overlap is shown in yellow in Figure 2; see also the 'Overlap' section in Table 1). Overlapping voxels included bilateral IPS and right angular gyrus (RAG), and they comprised roughly two-thirds (65.2\%) of all active voxels across both contrasts, indicating substantial co-modulation of neural responses by the two parameters. Several regions showed significant modulation for only one parameter, though it is possible that the other parameter also modulated neural responses, simply just below threshold. Note also that these results effectively constitute zero-order relations between each parameter (problem-size, interference) and neural activity. In the next section, we quantify the unique predictive capacity of each parameter within each of the regions identified here. 
Table 1: Region Details

\begin{tabular}{lcccccccccccc} 
& \multicolumn{4}{c}{ Overlap } & \multicolumn{4}{c}{ Problem-Size } & \multicolumn{4}{c}{ Interference } \\
Region & $\mathbf{x}$ & $\mathbf{y}$ & $\mathbf{z}$ & Volume & $\mathbf{x}$ & $\mathbf{y}$ & $\mathbf{z}$ & Volume & $\mathbf{x}$ & $\mathbf{y}$ & $\mathbf{z}$ & Volume \\
\hline LDLPFCp & -39 & 2 & 32 & 391 & -40 & 2 & 32 & 453 & -40 & 5 & 31 & 1085 \\
PreSMA & -1 & 11 & 47 & 1292 & -1 & 11 & 47 & 1324 & -1 & 10 & 46 & 3142 \\
LINSa & -32 & 18 & 8 & 829 & -32 & 18 & 8 & 849 & -31 & 18 & 9 & 1078 \\
LIPS & -30 & -52 & 42 & 3608 & -31 & -52 & 42 & 3967 & -30 & -53 & 42 & 6343 \\
RIPS & 27 & -56 & 44 & 240 & 27 & -57 & 43 & 299 & 27 & -55 & 44 & 899 \\
RAG & 47 & -56 & 27 & 669 & 47 & -56 & 27 & 871 & 47 & -56 & 27 & 966 \\
RCBM & 30 & -55 & -21 & 316 & 30 & -56 & -21 & 367 & 28 & -56 & -22 & 865 \\
\hline LACCd $^{1}$ & & & & & -7 & 20 & 34 & 220 & & & & \\
RMTG $^{1}$ & & & & & 57 & -13 & -6 & 344 & & & & \\
\hline LMFG $^{2}$ & & & & & & & & & -27 & -1 & 54 & 477 \\
LPMd $^{2}$ & & & & & & & & & -47 & -5 & 47 & 231 \\
LITGp $^{2}$ & & & & & & & & & -45 & -57 & -5 & 685 \\
LPUT $^{2}$ & & & & & & & & -17 & 7 & 5 & 759 \\
RPUT $^{2}$ & & & & & & & & & 18 & 8 & 4 & 420 \\
\hline
\end{tabular}

Note: 'Overlap' indicates overlapping voxels for a given ROI, where applicable. ${ }^{1}$ ROI showing a significant result only for problem-size at the whole-brain level; ${ }^{2} \mathrm{ROI}$ showing a significant result only for interference at the whole-brain level. LDPFCp = left posterior dorsolateral prefrontal cortex, LMFG = left middle frontal gyrus, LPMd, left dorsal premotor, PreSMA = presupplementary area, LINSa = left anterior insula, LACCd = left dorsal anterior cingulate cortex, LITGp = left posterior inferior temporal gyrus, RMTG = right middle temporal gyrus, LIPS = left intraparietal sulcus, RIPS = right intraparietal sulcus, RAG = right angular gyrus, LPUT = left putamen, RPUT $=$ right putamen, RCBM = right cerebellum. Volume is measured in $\mathrm{mm}^{3}$.

Figure 2: Regions showing modulation by Problem-Size and Interference

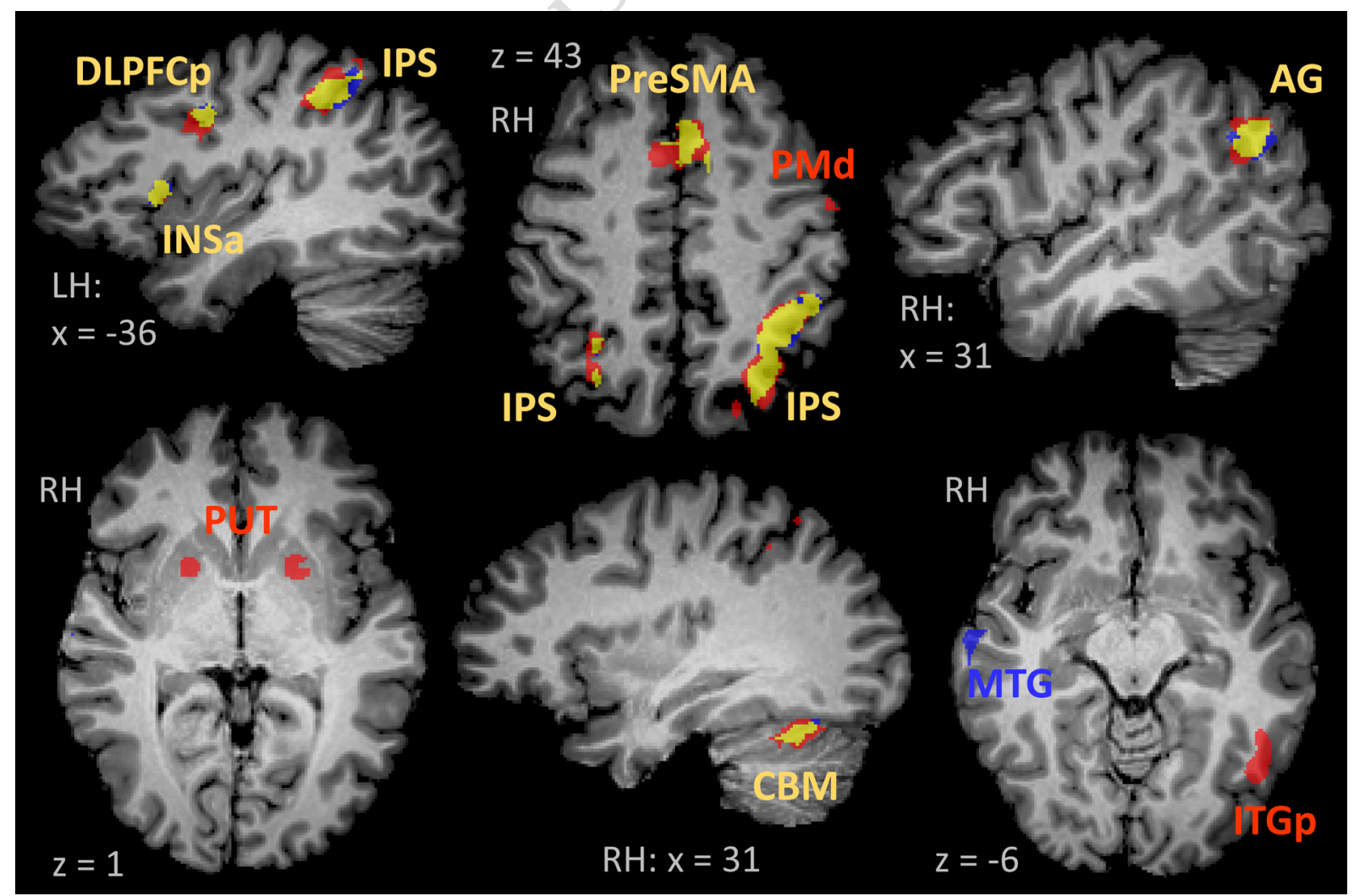


Figure 2 depicts regions significantly modulated by problem-size and/or interference at the whole-brain level. Note that the effects shown here are zero-order relations, in that effects of problem-size do not take into account interference, and vice versa. For unique effects, see Figure 3. Regions in blue are regions active for problem-size, regions in red are active for interference, and regions in yellow comprise overlapping voxels for both problem-size and interference. See Table 1 for complete region details and abbreviations.

\section{Unique Effects}

Zero-order effects in the previous section indicated substantial overlap in terms of brain areas modulated by both problem-size and interference. It is thus important to assess the unique contributions of each parameter within these regions to address the question of whether these contributions are unique or effectively one and the same. Moreover, while several regions showing significant modulation by just one parameter at the whole-brain level, it is important to address the concern that the other parameter modulated activity just below threshold, and hence whether, once one accounts for this potential sub-threshold modulation, the original effect still obtains. We address both issues in this section. Note that although some regions were overlapping (Table 1), there were nevertheless subtle differences in the voxels active for each parameter around the edges of these ROIs. To respect the possibility that these subtle differences may nevertheless prove key, we analyzed regions from the problem-size and interference whole-brain contrasts (Figure 2, Table 1) separately. Results for problem-size regions can be seen in Figure 3a; results for interference regions can be seen in Figure 3b.

Note that Figure 3 gives both zero-order effects and partial (unique) effects. The former are shown in lighter shade and labeled 'zero-order' in the figure legends. These effects are similar to zero-order correlations between the parametric predictor and neural activity, in that they do not control for the influence of the other parameter. As these are the effects identified by the whole-brain analysis, interpreting them in and of themselves would essentially constitute double-dipping. We provide them here (1) as visual confirmation of the whole-brain results, but more importantly (2) to provide a frame of reference for the main focus of Figure 3: the unique effects of each parameter. The unique effects are shown in slightly darker shade with bold outline (labeled as 'unique' in the figure legends). These effects constitute the fit between a given parameter and neural activity, controlling for (i.e., over and above) the influence of the other parameter. As expected, the unique effects tend to be smaller than the zero-order effects; the key, though, is in assessing which parameter - problem-size, interference, 
both or neither - retains significant unique predictive capacity of neural responses. Finally, note that effect-sizes are given in Figure 3 to aid generalizability across studies (raw means and standard-errors can be found in Table 2). Two statistical thresholds are given: the traditional $p<.05$ (dotted grey line) and the more conservative $p<.005$ (solid grey line). The latter roughly corresponds to Dunn-Šidák correction for multiple comparisons: $p=.0057$ in [a] (9 regions); $p=.0043$ in [b] (12 regions).

We first address overlap regions (yellow portions of Figure 3). We found significant unique modulation of neural activity by problem-size in 6 of the 7 overlap regions: LDLPFCp, PreSMA, LINSa, LIPS, RIPS and RAG. Indeed, most problem-size results were highly significant (obtaining at the more stringent significance threshold), and problem-size contribution was significantly greater than that of interference in 4 of the 7 overlap regions (denoted by a gold asterisk in Figure 3). Results were overall similar whether one looked at overlap regions defined by the problem-size (Figure 3a) or interference (Figure 3b) whole-brain contrasts. In contrast, we found significant unique modulation of neural activity by interference in only 3 of the 7 overlap regions: PreSMA, RAG and RCBM, each only at the more liberal threshold of $p<.05$ ). This suggests that the whole-brain overlap effects were largely driven by problem-size. That said, as with the behavioral results, additional unique variance in neural activity attributable to interference was seen in several regions, hence it would be premature to discount interference altogether. A final point is that modulation in all regions save one was positive, indicating greater positive deflection of neural activity as problem-size and/or interference increased; the RAG showed the opposite pattern.

The two 'problem-size' regions (blue portions of Figure 3) both showed significant effects of problem-size; LACCd also showed a significant effect of interference. Note also that RMTG, similar to RAG, showed negative effects, indicating significantly reduced activation as problem-size increased. Perhaps surprisingly, regions that, at the whole-brain level appeared to show only effects of interference (red portions of Figure 3) in fact tended to show somewhat larger unique effects of problem-size. While the zero-order effects of interference tended to be larger (albeit non-significantly so), each of these regions also showed a zero-order effect of problem-size (which was likely just below the critical whole-brain threshold). Crucially, taking into account these subthreshold effects revealed a somewhat different picture. While 3 of the 5 showed significant unique effects of problem-size (LMFG, LITGp, LPUT), only 2 of the 5 showed 
unique effects of interference (bilateral putamen), though it is important to note that the difference between effects reached significance only in LMFG. These results underscore two points: First, it is crucial to take into account potential competing (correlated) predictors, even if these predictors obtain only at subthreshold levels at the whole-brain level. Second, these regions conform to the broader pattern whereby problem-size accounts for a greater unique proportion of multiplication-related variance, with interference accounting for additional significant variance in a smaller set of regions.

\section{Figure 3: Unique neural effects of problem-size and interference}
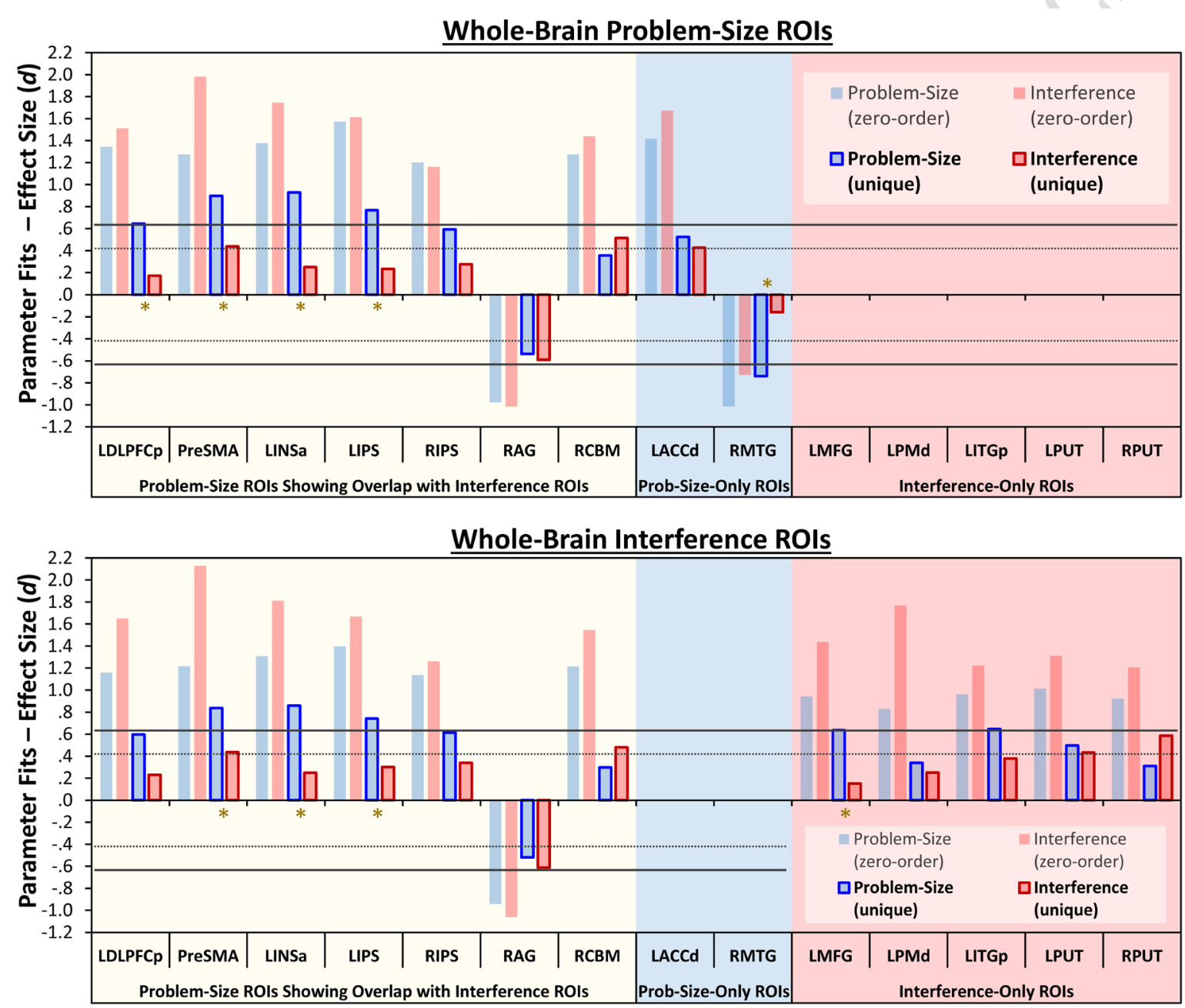

Figure 3 shows predictive effects of neural activity for problem-size and interference in [a] problem-size and [b] interference regions from the whole-brain analyses (Figure 2, Table 1). Effects are reported here as effect-sizes; means and standard errors are given in Table 2. Lighter shaded bars correspond to zero-order effects (the same as those used to identify the regions in the whole-brain analyses). They are provided here to give visual context to the unique effects, which are shown in darker bars in bold outline, which are the primary focus of this analysis. Regions in the yellow 'Overlap' sub-pane showed overlapping whole-brain effects. Regions from the separate contrasts were treated separately, though they are aligned here across sub-figures [a] and [b] to aid in visual comparison. The dotted grey line corresponds to $p<.05$; the solid grey line corresponds to the more conservative threshold of $p<.005$ (see text for details). 
Table 2: Means and standard-errors of neural effects

\begin{tabular}{|c|c|c|c|c|c|}
\hline \multicolumn{6}{|c|}{ [a] Whole-Brain Problem-Size ROIs } \\
\hline & ROI & $\begin{array}{l}\text { Problem-Size } \\
\text { (zero-order) }\end{array}$ & $\begin{array}{l}\text { Interference } \\
\text { (zero-order) }\end{array}$ & $\begin{array}{c}\text { Problem-Size } \\
\text { (unique) }\end{array}$ & $\begin{array}{c}\text { Interference } \\
\text { (unique) }\end{array}$ \\
\hline \multirow{7}{*}{ Overlap } & LDLPFCp & $.87(.13)$ & $.73(.10)$ & $.90(.28)$ & $.17(.21)$ \\
\hline & PreSMA & $.98(.16)$ & $.88(.09)$ & $.89(.20)$ & $.30(.14)$ \\
\hline & LINSa & $.89(.13)$ & $.75(.09)$ & $.86(.19)$ & $.17(.14)$ \\
\hline & LIPS & $.87(.11)$ & .75 (.10) & $.89(.24)$ & $.20(.18)$ \\
\hline & RIPS & $.66(.11)$ & $.61(.11)$ & $.55(.19)$ & $.24(.18)$ \\
\hline & RAG & $-.81(.17)$ & $-.55(.11)$ & $-.56(.21)$ & $-.39(.14)$ \\
\hline & RCBM & $.70(.11)$ & $.66(.09)$ & $.57(.33)$ & $.47(.19)$ \\
\hline \multirow{2}{*}{ Problem-Size } & $\mathrm{LACCd}$ & $.73(.10)$ & $.81(.10)$ & $.62(.24)$ & $.38(.18)$ \\
\hline & RMTG & $-.69(.14)$ & $-.37(.10)$ & $-.69(.19)$ & $-.09(.11)$ \\
\hline \multirow{5}{*}{ Interference } & LMFG & & & & \\
\hline & LPMd & & & & \\
\hline & LITGp & & & & \\
\hline & LPUT & & & & \\
\hline & RPUT & & & & \\
\hline
\end{tabular}

\begin{tabular}{|c|c|c|c|c|c|}
\hline \multicolumn{6}{|c|}{ [b] Whole-Brain Interference ROIs } \\
\hline & & $\begin{array}{c}\text { Problem-Size } \\
\text { (zero-order) }\end{array}$ & $\begin{array}{l}\text { Interference } \\
\text { (zero-order) }\end{array}$ & $\begin{array}{l}\text { Problem-Size } \\
\text { (unique) }\end{array}$ & $\begin{array}{l}\text { Interference } \\
\text { (unique) }\end{array}$ \\
\hline \multirow{7}{*}{ Overlap } & LDLPFCp & $.82(.14)$ & $.71(.09)$ & $.80(.27)$ & $.21(.18)$ \\
\hline & PreSMA & $.84(.14)$ & $.78(.07)$ & $.77(.19)$ & $.26(.12)$ \\
\hline & LINSa & $.86(.13)$ & $.75(.08)$ & $.86(.20)$ & $.16(.13)$ \\
\hline & LIPS & $.80(.12)$ & $.74(.09)$ & $.79(.22)$ & $.24(.16)$ \\
\hline & RIPS & $.61(.11)$ & $.59(.10)$ & $.46(.15)$ & $.28(.17)$ \\
\hline & RAG & $-.80(.17)$ & $-.55(.11)$ & $-.55(.21)$ & $-.41(.14)$ \\
\hline & RCBM & $.61(.10)$ & $.63(.08)$ & $.48(.33)$ & $.46(.20)$ \\
\hline \multirow{2}{*}{ Problem-Size } & LACCd & & & & \\
\hline & RMTG & & & & \\
\hline \multirow{5}{*}{ Interference } & LMFG & $.62(.13)$ & $.65(.09)$ & $.70(.22)$ & $.12(.16)$ \\
\hline & LPMd & $.47(.12)$ & $.47(.05)$ & $.53(.32)$ & $.19(.15)$ \\
\hline & LITGp & $.60(.13)$ & $.61(.10)$ & $.56(.18)$ & $.27(.15)$ \\
\hline & LPUT & $.47(.10)$ & $.55(.09)$ & $.39(.16)$ & $.28(.13)$ \\
\hline & RPUT & $.49(.11)$ & $.52(.09)$ & $.29(.19)$ & $.42(.15)$ \\
\hline
\end{tabular}

Table 2 gives means (standard errors) for Problem-Size and Interference effects in each ROI, averaged across participants. Effect-sizes comparing these values against 0 are given in Figure 3. 


\section{DISCUSSION}

Arithmetic is a quintessential mathematical ability used by many children and adults on a more or less daily basis. Mental multiplication is one of the most common forms of arithmetic, which is thought to be primarily solved via direct retrieval from memory. The key factors that govern memory retrieval in arithmetic - and in particular retrieval of multiplication facts - remain a major source of interest for researchers in the domain of numerical cognition, and more broadly in the domain of memory retrieval as well. Two of the main factors thought to influence multiplication memory retrieval both behaviorally and neurally are problem-size (e.g., Stazyk, Ashcraft, \& Hamann, 1982) and interference (De Visscher \& Noël, 2014a,b; De Visscher et al., 2015). However, because these two factors are often inter-twined, here we sought to identify the unique influences of problem-size and interference on both performance and neural responses during multiplication fact-retrieval in healthy adults. Behavioral results showed that both problem-size and interference explained separate unique portions of responsetime variance, but with a significantly stronger contribution from problem-size. Wholebrain fMRI results using a paradigm that isolated multiplication fact-retrieval from response-selection showed highly overlapping brain areas parametrically modulated by both problem-size and interference. Within these regions, problem-size was the stronger and more consistent unique modulating factor. This result was obtained in both overlapping regions as well as those that appeared to respond only to problem-size or interference at the whole-brain level, thus underscoring the need to look beyond anatomical overlap and arbitrary thresholds by accounting for unique modulatory contributions. Problem-size, relative to interference, appears to be the more dominant factor in driving both behavioral and neural responses during multiplication factretrieval in adults. That said, additional unique contributions of interference (beyond problem-size) were identified in right angular gyrus and several subcortical regions associated with procedural processing. Hence, the unique contributions of both factors demonstrate the importance of considering the overlapping and unique contributions of each in explaining the cognitive and neural basis of mental multiplication.

One of the key features of the current study is that we allowed problem-size and interference to co-vary across trials, which is what in turn allowed us to assess both overlapping and unique contributions of each in a single study. Previous fMRI work looking at problem-size and interference pre-selected trials so as to create an 
orthogonal Problem-Size $\times$ Interference design (De Visscher et al., 2015, 2018). Such an approach is highly useful for isolating specific effects, as was the intent of the authors of the papers in question. However, such an approach (1) a priori assumes that problemsize and interference indeed comprise independent factors, and (2) it thus does not allow one to explicitly test the validity of this assumption across a broader array of commonly encountered problems.

By adopting a parametric approach, we were able to do precisely this. On the one hand, our results support the validity of this assumption. The behavioral data showed highly significant unique contributions of both problem-size and interference with respect to predicting multiplication response-times (Figure 1). That said, problem-size captured more unique behavioral variance. That is, the average standardized fit across subjects (mean partial- $r$ ) was nearly twice as large for problem-size as that seen for interference (.346 vs .194). Interestingly, this is roughly the opposite of what De Visscher et al. (2016) found when using a similar approach: those authors found significant unique effects of both parameters on response-times at the individual level, but somewhat stronger for interference rather than problem-size $(t \mathrm{~s}=4.4$ vs 2.5 , respectively). One reason for this difference in results is that De Visscher et al. examined multiplication performance in $4^{\text {th }}$ grade children. The interference parameter was initially conceived within a developmental framework as a means of understanding the relative ease and difficulty with which individuals acquire different multiplication facts, in particular capturing the order in which these facts are learned in primary school. Hence, mechanisms of proactive interference may exert a greater influence on performance when children are first acquiring and hence still practicing multiplication tables. In our case, we examined college-level adults whose age and education likely led most to have highly practiced retrieval access to the majority of the basic multiplication tables ( $2 \times 2$ through $9 \times 9)$. Repeated practice has long been understood to be an important means of overcoming proactive interference (e.g., Underwood \& Ekstrand, 1967), which is postulated to be the key mechanism at the heart of interference effects in arithmetic (De Visscher \& Noël, 2014a). Repeated practice, therefore, may have shifted the critical factor from interference to problem-size over development. Broadly, consistent with this idea, De Visscher and Noël (2014b) found that problem-size tended to be the slightly stronger predictor of multiplication response-times in adults and interference tended to be the slightly stronger predictor of multiplication response- 
times in $3^{\text {rd }}$ and $5^{\text {th-grade }}$ children. However, some caution is needed, as these effects were not significantly different from one another, which may have been in part due to the fact that data were analyzed at the group-level (ignoring subject-level variability).

That said, from a broader theoretical perspective, smaller quantities are encountered more frequently (Dehaene \& Mehler, 1992), thus affording more opportunities for retrieval practice, which in turn should lead to faster retrieval times. This frequency-based account of the problem-size effect is supported by a recent paper that used fMRI data to distinguish between competing cognitive accounts of the problem-size effect in multiplication (tiberghien et al., under review). Using a representational similarity analysis approach, the authors found that the neural patterns elicited by larger problems (6-9) were more similar to one another than were the neural patterns elicited by smaller problems (1-4), indicating that larger problems are represented less distinctly from one another, and smaller problems are represented more distinctly from one another.

In sum, though the difference between the current study and De Visscher et al.'s (2016) results are intriguing from a developmental perspective, some caution is warranted. A longitudinal study would be the gold-standard for drawing developmental inferences. Furthermore, an important methodological point is that De Visscher et al. recorded responses by having children type their answers on a keypad, whereas we used vocal responses (with trial repetitions to circumvent missed trials due to technical concerns when using a voice-key). Using a keypad is not problematic in its own right, and, may in some circumstances even be desirable. However, some trials will involve typing the same keys (e.g., $3 \times 8$ and $4 \times 7$ will both involve typing a 2 ), which should make response-times on such trials more similar (because the motor actions are more similar). Because the interference parameter is defined based on the number of overlapping digits, it will also predict similar performance on trials that involve typing the same digit. It may thus have been the case that the use of the typing response modality inflated the apparent correlation between response-times and interference. Because we used vocal responses here, this may explain the relatively smaller relation we observed with interference. Regardless, future work examining the developmental time-course of the relative influence of problem-size and interference on multiplication performance warrants future study. 
In the present study, we also found substantial evidence that problem-size, relative to interference, tended to be the overall stronger unique predictor of neural responses during multiplication fact-retrieval. Significant unique effects of problem-size were found in 8 of the 9 regions that showed a (zero-order) effect of problem-size at the whole-brain level (Figure 3a), and 9 of the 12 regions that showed a (zero-order) effect of interference at the whole-brain level (Figure 3b). By contrast, interference showed significant unique effects in just 4 of 9 and 5 of 12 regions, respectively. Moreover, problem-size effects were stronger on average in every region except one (RPUT), with this difference obtaining significance in several prefrontal and parietal regions, as well as RMTG. The broader pattern thus appears to corroborate the behavioral results: problem-size accounts for a somewhat greater unique proportion of multiplicationrelated neural activity, albeit with interference accounting for additional significant variance in a smaller set of regions. As such, these results also support the notion that, at least in educated adults, years of experience and exposure to the size-related frequencygradients with which one encounters problems of varying size may well be the predominant factor in multiplication processing not just behaviorally, but at the neural level as well.

It is important to note that this convergence of behavioral and neural results is made more remarkable by the fact that the fMRI analyses focused exclusively on portions of the task that were isolated from the generation of specific behavioral responses. Hence, we argue that these results are most likely indicative of multiplication retrieval, as opposed to response selection. By extension, potential explanations of our results referring to 'difficulty' would need to carefully specify just what is meant by 'difficulty'. At the neural level, it would need to refer to a process that occurs prior to response-selection. Moreover, it would need to propose mechanisms that could explain the unique effects of problem-size and interference respectively; that is, a general explanation would struggle to account for both effects as each comprises unique aspects of behavioral and neural responses. Instead, we suggest that problem-size and interference comprise disparate influences on mental arithmetic. On the one hand these influences overlap with one another (both in terms of variance explained and the specific anatomical regions involved). On the other hand, we show here that these influences can nevertheless be decomposed into disparate sources (thus also 
highlighting the importance of taking into account potential competing predictors, even if these predictors obtain only at sub-threshold levels at the whole-brain level).

More specifically, we propose that problem-size primarily reflects repeated exposure to multiplication problems as a function of underlying numerical frequencies. Consistent with this, unique problem-size effects tended to be strongest in prefrontal and temporal regions associated with control of retrieval (e.g., Wagner et al., 2001; Badre et al., 2005; Whitney et al., 2012; Davey et al., 2015; Khader et al., 2015), as well as parietal regions associated with numerical representation (e.g., Sokolowski et al., 2017). The majority ( 4 of 6 ) of unique interference effects appeared in subcortical regions associated with procedural memory and complex motor control (bilateral putamen, cerebellum, Pre-SMA; Packard \& Knowlton, 2002; Lalonde \& Strazielle, 2003; Nachev et al., 2007). As proactive interference has long been known to operate on procedural (i.e., non-declarative) memory (e.g., Lustig et al., 2001; Robertson, 2004), we suggest this may constitute a previously overlooked source of interference effects in multiplication. Further evidence consistent with this view is that interference effects appear to exert a stronger influence on results (at least relative to problem-size) in paradigms that either emphasize motor responses (such as typing on a keyboard; De Visscher et al., 2016) or include motor responses in the neural signal being examined (e.g., De Visscher et al., 2015, 2018). Furthermore, LeFevre et al. (1996) reported that non-retrieval-based procedural strategies may be used as much as $20 \%$ of the time even for single-digit multiplication in adults. These strategies include repetitive, recursive processing, such as repeated addition and recitation of multiplication series (5..10..15 ...) which are similar in spirit to the embedded manner in which the interference parameter is defined here (following De Visscher and colleagues). Efficiency in repetitive processing is one hallmark of procedural memory; hence, it may be that interference indexes the proficiency with which individuals implement these non-retrieval strategies, which in turn rely on procedural memory processes.

Thus, we suggest that problem-size captures primarily retrieval-based memory influences on multiplication processing, and interference captures primarily procedural memory influences (e.g., those directly related to generating over-learned motor outputs) on multiplication processing (for a discussion of the distinction between these memory systems and their potential role in understanding the neuro-cognitive bases of mathematical processing in general, see Evans \& Ullman, 2016). However, our 
interpretation in the preceding paragraph relies at least in part on reverse inference; hence, this proposal must at present remain tentative, though we encourage testing this hypothesis as a potentially useful impetus for future research.

Along these lines, another lingering question concerns the fact that right angular gyrus (RAG) showed significant unique effects of both problem-size and interference. Previous work has shown significant interference effects in the angular gyrus (De Visscher et al., 2018; note also that De Visscher et al., 2015, found a similar result, but in the left hemisphere) ${ }^{3}$, and substantial work has shown angular gyrus activity to be associated with arithmetic processing in general (e.g., Arsiladou \& Taylor, 2011; Grabner et al., 2013; Bloechle et al., 2016). This region may thus represent an important confluence of the respective influences of problem-size and interference on multiplication processing. On the other hand, the purported functions of the angular gyrus are many (Seghier, 2013), so further work is needed to disentangle the potential mechanisms by which this confluence may arise.

A final piece of the puzzle is that, while we identified unique effects, we also found evidence for overlapping influences of problem-size and interference in both brain and behavior (with the latter result being consistent with De Visscher et al., 2014b, 2016). That is, the unique effects were notably smaller than the zero-order effects. One can see this in the smaller bold bars relative to the larger faded bars in Figures 1 and 2. This implies that size and interference effects on multiplication do contain a shared component. Though outside the scope of the current paper, an interesting question for future work may be to understand the reasons for this overlap both at the behavioral and the neural level.

\section{Conclusion}

In sum, we show that the unique influence of problem-size on behavioral and neural responses during retrieval of multiplication facts tends to outweigh that of interference. However, significant unique effects of each remain. Broadly, we proposed that problemsize effects are driven largely by exposure to frequency gradients, which may thus be of greater influence than interference only later in development. Such instance-based

\footnotetext{
3 We found a negative effect in the current study (greater interference - and problem-size - predicted greater reduction in neural responses), which is consistent with what De Visscher et al. $(2015,2018)$ reported previously. It is also consistent with work on arithmetic processing in general, which has typically found significant deactivation in the angular gyrus during arithmetic processing (see, e.g., Grabner et al., 2013, for an extensive discussion).
} 
memory effects may in turn be linked more strongly to declarative memory systems. We also propose that interference effects may be tied more closely to proactive interference effects in procedural memory that are more easily detected either in paradigms emphasizing motor responses or populations, such as children, whose grasp of multiplication facts may still be developing (i.e., not yet progressed to the point of direct retrieval). Together, these results provide unique insight into multiplication processing - a quotidian and quintessential example of memory retrieval in humans.

\section{REFERENCES}

Arsalidou, M., \& Taylor, M. J. (2011). Is 2+ 2=4? Meta-analyses of brain areas needed for numbers and calculations. Neuroimage, 54(3), 2382-2393.

Ashcraft, M. H. (1987). Children's Knowledge of simple arithmetic. A developmental model and simulation. In C. J. Brainerd, R. Kail, \& J. Bisanz (Eds.) Formal methods in developmental research (pp. 302-338). New York: Springer-Verlag.

Ashcraft, M. H., \& Christy, K. S. (1995). The frequency of arithmetic facts in elementary texts: Addition and multiplication in grades 1-6. Journal for Research in Mathematics Education, 396-421.

Ashcraft, M. H., \& Guillaume, M. M. (2009). Mathematical cognition and the problem-size effect. Psychology of learning and motivation, 51, 121-151.

Badre D, Poldrack RA, Paré-Blagoev EJ, Insler RZ, Wagner AD (2005). Dissociable controlled retrieval and generalized selection mechanisms in ventrolateral prefrontal cortex. Neuron, 47(6):907-18.

Bloechle J, Huber S, Bahnmueller J, Rennig J, Willmes K, Cavdaroglu S, Moeller K, Klein E (2016). Fact learning in complex arithmetic-the role of the angular gyrus revisited. Hum Brain Mapp, 37(9):3061-79.

Campbell, J. I. D. (1995). Mechanisms of simple addition and multiplication: A modified network-interference theory and simulation. Mathematical cognition, 1(2), 121-164.

Campbell, J. I. D. (1997). On the relation between skilled performance of simple division and multiplication. Journal of Experimental Psychology: Learning, Memory, and Cognition, 23, 1140-1159.

Campbell, J. I. D. (Ed.). (2005). Handbook of mathematical cognition. New York: Psychology Press.

Campbell, J. I. D., \& Xue, Q. (2001). Cognitive arithmetic across cultures. Journal of Experimental Psychology: General, 130(2), 299.

Davey J, Cornelissen PL, Thompson HE, Sonkusare S, Hallam G, Smallwood J, Jefferies E (2015). Automatic and Controlled Semantic Retrieval: TMS Reveals Distinct Contributions of Posterior Middle Temporal Gyrus and Angular Gyrus. J Neurosci, 35(46):15230-9. 
Dehaene S \& Mehler J (1992). Cross-linguistic regularities in the frequency of number words. Cognition, 43(1), pp.1-29.

De Visscher A, Noël MP (2014a). Arithmetic facts storage deficit: The hypersensitivity-tointerference in memory hypothesis. Developmental Science, 17(3), 434-442.

De Visscher A, Noël MP (2014b). The detrimental effect of interference in multiplication facts storing: Typical development and individual differences. Journal of Experimental Psychology: General, 143(6):2380.

De Visscher, A., Berens, S. C., Keidel, J. L., Noël, M. P., \& Bird, C. M. (2015). The interference effect in arithmetic fact solving: an fMRI study. NeuroImage, 116, 92-101.

De Visscher A, Noël MP, De Smedt B (2016). The role of physical digit representation and numerical magnitude representation in children's multiplication fact retrieval. $J$ Exp Child Psychol, 152:41-53.

De Visscher A, Vogel SE, Reishofer G, Hassler E, Koschutnig K, De Smedt B, Grabner RH (2018). Interference and problem size effect in multiplication fact solving: Individual differences in brain activations and arithmetic performance. NeuroImage, 172:718727.

Evans TM, Ullman MT (2016). An Extension of the Procedural Deficit Hypothesis from Developmental Language Disorders to Mathematical Disability. Front Psychol, 7:1318.

Fayol, M., \& Thevenot, C. (2012). The use of procedural knowledge in simple addition and subtraction problems. Cognition, 123(3), 392-403.

Forman, S. D., Cohen, J. D., Fitzgerald, M., Eddy, W. F., Mintun, M. A., \& Noll, D. C. (1995). Improved assessment of significant activation in functional magnetic resonance imaging (fMRI): use of a cluster-size threshold. Magnetic Resonance in medicine, 33(5), 636-647.

Grabner, R. H., Ansari, D., Koschutnig, K., Reishofer, G., \& Ebner, F. (2013). The function of the left angular gyrus in mental arithmetic: evidence from the associative confusion effect. Human brain mapping, 34(5), 1013-1024.

Khader PH, Pachur T, Weber LA, Jost K (2016). Neural Signatures of Controlled and Automatic Retrieval Processes in Memory-based Decision-making. J Cogn Neurosci, 28(1):69-83.

Kirk, E. P., \& Ashcraft, M. H. (2001). Telling stories: the perils and promise of using verbal reports to study math strategies. Journal of Experimental Psychology: Learning, Memory, and Cognition, 27(1), 157.

Lalonde R, Strazielle C (2003). The effects of cerebellar damage on maze learning in animals. Cerebellum, 2(4):300-9.

LeFevre JA, Bisanz J, Daley KE, Buffone L, Greenham SL, Sadesky GS (1996). Multiple routes to solution of single-digit multiplication problems. Journal of Experimental Psychology: General, 125(3):284.

Lustig C, Hasher L (2001). Implicit memory is vulnerable to proactive interference. Psychol Sci, 12(5):408-12.

McCloskey, M., \& Lindemann, A. M. (1992). MATHNET: Preliminary results from a distributed model of arithmetic fact retrieval. Advances in psychology, 91, 365-409. 
Nachev P, Wydell H, O'neill K, Husain M, Kennard C (2007). The role of the presupplementary motor area in the control of action. NeuroImage, 36, Suppl 2:T155-63.

Nairne, J. S. (1990). A feature model of immediate memory. Memory \& Cognition, 18(3), 251-269.

Oberauer, K., \& Lange, E. B. (2008). Interference in verbal working memory: Distinguishing similarity-based confusion, feature overwriting, and feature migration. Journal of Memory and Language, 58(3), 730-745.

Robertson EM (2004). Skill learning: putting procedural consolidation in context. Curr Biol, 14(24):R1061-3.

Seghier ML (2013). The angular gyrus: multiple functions and multiple subdivisions. Neuroscientist, 19(1):43-61.

Siegler, R. S., \& Shrager, J. (1984). Strategy choices in addition and subtraction: How do children know what to do. Origins of cognitive skills, 23(1), 229-293.

Sokol, S. M., McCloskey, M., Cohen, N. J., \& Aliminosa, D. (1991). Cognitive representations and processes in arithmetic: Inferences from the performance of brain-damaged subjects. Journal of Experimental psychology: Learning, memory, and Cognition, 17(3), 355.

Sokolowski HM, Fias W, Mousa A, Ansari D (2016). Common and distinct brain regions in both parietal and frontal cortex support symbolic and nonsymbolic number processing in humans: A functional neuroimaging meta-analysis. Neurolmage, 146:376-394.

Stazyk, E. H., Ashcraft, M. H., \& Hamann, M. S. (1982). A network approach to mental multiplication. Journal of Experimental Psychology: Learning, Memory, and Cognition, $8(4), 320$.

Talairach, J., \& Tournoux, P. (1988). Co-planar stereotaxic atlas of the human brain. 3Dimensional proportional system: an approach to cerebral imaging.

Tieberghien K, De Smedt B, Fias W, Lyons IM (under review). Distinguishing between Cognitive Explanations of the Problem Size Effect in Mental Arithmetic via Representational Similarity Analysis of fMRI Data.

Underwood, B. J., \& Ekstrand, B. R. (1967). Studies of distributed practice: XXIV. Differentiation and proactive inhibition. Journal of Experimental Psychology, 74(4, Pt.1), 574-580.

Verguts, T., \& Fias, W. (2005). Interacting neighbors: A connectionist model of retrieval in single-digit multiplication. Memory \& Cognition, 33(1), 1-16.

Wagner AD, Paré-Blagoev EJ, Clark J, Poldrack RA (2001). Recovering meaning: left prefrontal cortex guides controlled semantic retrieval. Neuron, 31(2):329-38.

Whitney C, Kirk M, O'Sullivan J, Lambon Ralph MA, Jefferies E (2012). Executive semantic processing is underpinned by a large-scale neural network: revealing the contribution of left prefrontal, posterior temporal, and parietal cortex to controlled retrieval and selection using TMS. J Cogn Neurosci, 24(1):133-47. 


\section{APPENDIX A}

Table A-1: Problem-size and Interference Parameters per Problem

\begin{tabular}{|c|c|c|c|}
\hline Problem & Commutative pair & $\begin{array}{c}\text { Problem size } \\
\text { (product) }\end{array}$ & Interference parameter \\
\hline $2 \times 2$ & & 4 & 0 \\
\hline $2 \times 3$ & $3 \times 2$ & 6 & 0 \\
\hline $2 \times 4$ & $4 \times 2$ & 8 & 1 \\
\hline $2 \times 5$ & $5 \times 2$ & 10 & 0 \\
\hline $2 \times 6$ & $6 \times 2$ & 12 & 3 \\
\hline $2 \times 7$ & $7 \times 2$ & 14 & 4 \\
\hline $2 \times 8$ & $8 \times 2$ & 16 & 7 \\
\hline $2 \times 9$ & $9 \times 1$ & 18 & 7 \\
\hline $3 \times 3$ & & 9 & 0 \\
\hline $3 \times 4$ & $4 \times 3$ & 12 & 10 \\
\hline $3 \times 5$ & $5 \times 3$ & 15 & 2 \\
\hline $3 \times 6$ & $6 \times 3$ & 18 & 8 \\
\hline $3 \times 7$ & $7 \times 3$ & 21 & 13 \\
\hline $3 \times 8$ & $8 \times 3$ & 24 & 13 \\
\hline $3 \times 9$ & $9 \times 3$ & 27 & 9 \\
\hline $4 \times 4$ & & 16 & 5 \\
\hline $4 \times 5$ & $5 \times 4$ & 20 & 8 \\
\hline $4 \times 6$ & $6 \times 4$ & 24 & 12 \\
\hline $4 \times 7$ & $7 \times 4$ & 28 & 17 \\
\hline $4 \times 8$ & $8 \times 4$ & 32 & 25 \\
\hline $4 \times 9$ & $9 \times 4$ & 36 & 9 \\
\hline $5 \times 5$ & & 25 & 3 \\
\hline $5 \times 6$ & $6 \times 5$ & 30 & 6 \\
\hline $5 \times 7$ & $7 \times 5$ & 35 & 7 \\
\hline $5 \times 8$ & $8 \times 5$ & 40 & 9 \\
\hline $5 \times 9$ & $9 \times 5$ & 45 & 6 \\
\hline $6 \times 6$ & & 36 & 4 \\
\hline $6 \times 7$ & $7 \times 6$ & 42 & 22 \\
\hline $6 \times 8$ & $8 \times 6$ & 48 & 11 \\
\hline $6 \times 9$ & $9 \times 6$ & 54 & 13 \\
\hline $7 \times 7$ & & 49 & 7 \\
\hline $7 \times 8$ & $8 \times 7$ & 56 & 9 \\
\hline $7 \times 9$ & $9 \times 7$ & 63 & 17 \\
\hline $8 \times 8$ & & 64 & 19 \\
\hline $8 \times 9$ & $9 \times 8$ & 72 & 19 \\
\hline $9 \times 9$ & & 81 & 6 \\
\hline
\end{tabular}

